\title{
Can wearable haptic devices foster the embodiment of virtual limbs?
}

This is the peer reviewed version of the following article:

Original:

Frohner, J., Salvietti, G., Beckerle, P., Prattichizzo, D. (2019). Can wearable haptic devices foster the embodiment of virtual limbs?. IEEE TRANSACTIONS ON HAPTICS, 12(3), 339-349

[10.1109/TOH.2018.2889497].

Availability:

This version is availablehttp://hdl.handle.net/11365/1067210

since 2019-11-13T16:05:45Z

\section{Published:}

DOI:10.1109/TOH.2018.2889497

Terms of use:

Open Access

The terms and conditions for the reuse of this version of the manuscript are specified in the publishing policy. Works made available under a Creative Commons license can be used according to the terms and conditions of said license.

For all terms of use and more information see the publisher's website.

(Article begins on next page) 


\title{
Can wearable haptic devices foster the embodiment of virtual limbs?
}

\author{
Jakob Fröhner, Gionata Salvietti, Member, IEEE, Philipp Beckerle, Member, \\ IEEE, and Domenico Prattichizzo, Fellow, IEEE
}

\begin{abstract}
Increasing presence is one of the primary goals of virtual reality research. A crucial aspect is that users are capable of distinguishing their self from the external virtual world. The hypothesis we investigate is that wearable haptics play an important role in the body experience and could thereby contribute to the immersion of the user in the virtual environment. A within-subject study ( $n=32$ ) comparing the embodiment of a virtual hand with different implementations of haptic feedback (force feedback, vibrotactile feedback, and no haptic feedback) is presented. Participants wore a glove with haptic feedback devices at thumb and index finger. They were asked to put virtual cubes on a moving virtual target. Touching a virtual object caused vibrotactile-feedback, force-feedback or no feedback depending on the condition. These conditions were provided both synchronously and asynchronously. Embodiment was assessed quantitatively with the proprioceptive drift and subjectively via a questionnaire. Results show that haptic feedback significantly improves the subjective embodiment of a virtual hand and that force feedback leads to stronger responses to certain subscales of subjective embodiment. These outcomes are useful guidelines for wearable haptic designer and represent a basis for further research concerning human body experience, in reality, and in virtual environments.
\end{abstract}

Index Terms - wearable haptics, embodiment, haptics in VR, tactile feedback, virtual hand illusion.

\section{INTRODUCTION}

Virtual reality (VR) developers primarily aim at augmenting the user's sense of presence. A significant part of the research toward an improvement of presence is centered on sensory stimulation, avatar mobility, and avatar representativeness [1]. Biocca et al. argued that humans perceive the physical world in relation to their bodies, and therefore what we know about the world is constructed from patterns of energy detected by the body [2]. The implications of this theory for VR and interfaces designers is that virtual environment can be incorporated into user's reality if the system is able to provide affordances for users so that they may become embodied, or take some form or shape within the VR. In other words, the user is immersed in the virtual reality environment whether changes in the VR affect the user (cognitively, emotionally, and/or physiologically), and the user can affect the VR (interact with objects, etc.) [3]. In this regard, experience of bodily self in the $\mathrm{VE}$ is of paramount

- G. Salvietti and D. Prattichizzo are with the Department of Information Engineering and Mathematics, University of Siena, Siena, Italy.

- J. Fröhner is with the Chair of Information-oriented Control, Technical University of Munich, Munich, 80333 Germany

- P. Beckerle is with the Elastic Lightweight Robotics Group, Robotics Research Institute, Technische Universität Dortmund, Dortmund, 44221 Germany

- P. Beckerle is also with the Institute for Mechatronic Systems in Mechanical Engineering, Technical University of Darmstadt, Darmstadt, Germany.

- G. Salvietti and D. Prattichizzo are also with the Deparment of Advanced Robotics, Istituto Italino di Tecnologia, Genoa, Italy.

- This research has received funding from the European Union Seventh Framework Programme FP7/2007-2013 under grant no. 601165 of the project WEARHAP - WEARable HAPtics for humans and robots and EU H2020 funded project SoftPro (no. 688857) as well as the DFG project "Users' Body Experience and Human-Machine Interfaces in (Assistive) Robotics" (no. BE5729/3E11).

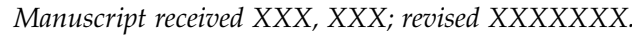

importance [4].

Haptic feedback is an increasingly popular affordance in virtual environments [5]. Force feedback has been introduced and studied for teleoperation purposes and it is now available in several simulators and gaming interfaces [6]. More sophisticated implementations are also popular, as researchers seek to increase users' presence and body experience. Sallnäs et al. [7] found that implementing a force feedback mechanism had positive effects on physical presence and task performance. Similarly, haptic feedback showed positive effects on embodiment in VR [8].

However, most of the experiments involving haptic feedback were realized either limiting haptics to the end effector of an instrument which was manipulated by the human user [8], [9], or with the use of exoskeleton mechanically controlled to impart forces on the user according to the user interaction in the virtual environment [10]. These solutions highly constrained the user possible interaction to the relative reduced workspaces of the devices. Only recently, studies on the modulation of ownership and agency in the virtual hand illusion have been performed using wearable technologies. For instance, in [11], a wearable vibrotactile system has been used to study when feelings of ownership and agency can be achieved in immersive virtual environments, when the participant is realistically interacting and performing natural upper limb movements. Wearable haptics [12] allows a hand free operation and thus make possible focusing on how tactile stimuli can affect the embodiment in VR.

In this study, different types of haptic feedback modalities have been compared while manipulating objects in a virtual environment. Even though the virtual hand does not belong to one's own body, this study focuses on how to induce the illusion that the virtual hand belongs to the person 
that acts in the virtual environment. We investigate possible modulations of ownership, under different sensorimotor conditions: (i) force feedback achieved through wearable active thimbles able to provide forces in the direction normal to the fingertip pulp [13], (ii) vibrotactile stimulation at the fingertip, and (iii) no haptic feedback.

We hypothesize that the experience of embodying the virtual hand increases if haptic feedback is provided. In this, differences between force and vibrotactile feedback are of specific interest to provide directions for the design of haptic feedback devices. Moreover, delaying the virtual hand is hypothesized to decrease the experience of embodiment significantly. We prove through a behavioral questionnaire measurements a significant higher sensation of embodying the virtual hand when haptic feedback is provided. Besides, delaying the virtual hand by $350 \mathrm{~ms}$, the embodiment decreases significantly. The proposed framework can potentially be exploited in applications for training, rehabilitation, gaming and entertainment, and represents a powerful tool for further studies of body perception and awareness.

\section{Related Works}

\subsection{Human body experience}

Having the experience that an external hand is part of one's own body was first investigated in the "Rubber Hand Illusion" (RHI) experiment [14]. One hand of the participants was placed on a table, but hidden from the participants' view. The experimenter stroked the participants' hidden hand and a visible rubber hand simultaneously. The participants of the study reported that they experienced the rubber hand as a part of their body. They reported to feel the touch on the visible rubber hand rather than their hidden real one. This phenomenon occurs due to multisensory integration of vision, touch, and proprioception, while the brain tries to combine all information and finally moves the perceived sensation from the real hand to the position of the rubber hand since participants can only see the stroke on the rubber hand [15].

Slater et al. [4] showed that the body ownership illusion can be induced through computer-generated visuotactile sensory stimulations within immersive VR scenarios, under static conditions. In their experiment, participants saw in stereo a virtual arm being continuously touched by a virtual ball, at the time that the experimenter stroked in synchronicity the participant's hand with a real ball. In [16], the authors tested the Virtual Hand Illusion (VHI) under different conditions that included self-stimulation with the left hand and passive and active movements through a haptic device. Through self-reported questionnaires, they found that the subjective illusion of ownership of the virtual hand occurred when either synchronous visuomotor or visuotactile stimulations were provided, and that active movements of the hand elicited stronger feelings of ownership than passive movements. They perceive body ownership for the rubber hand, which means they perceive the rubber hand incorporated to their own body [17]. Longo et al. investigated a principal component analysis of 27 questionnaire statements to develop the major components of the embodiment. This analysis revealed three subcomponents of the embodiment of rubber hand: ownership, location, and agency [18].

The experimental setup of the rubber hand illusion can be extended using virtual or robotic hands [4], [19], [20], [21], [22], [23]. Virtual reality techniques provide a promising method to study body representation and its perception, allowing the simulation of conditions that cannot be reproduced in the real world [24], [25] . During the last few years, several studies have successfully reproduced the RHI within virtual environments (VE), eliciting the virtual hand illusion. In the VHI setups, the participants perceive body ownership for an external hand since it moves synchronously with the participant's real but covered hand [26]. IJsselsteijn et al. [27] enabled a VHI by stroking a 2D video image of a hand projected on a table, and compared the strength of the illusion with respect to the original RHI. However, they found that the unmediated condition produced the strongest illusion, as indicated by self-reports and proprioceptive drift towards the rubber hand compared to the virtual hand illusion [27]. The use of a tele-operated robotic hand as the rubber hand has been shown to elicit a much stronger RHI when compared with the classical rubber hand paradigm, probably since participants experience themselves as agents of the rubber hand movement [21]. Beckerle et al. analyzed technical design requirements that concern the occurrence and quality of the illusion [28] to induce a substantial robotic hand illusion. They characterized hiding the real limb, anatomical plausibility, visual appearance, temporal delay, and software controlled experimental conditions as most important. These guidelines can also inspire the development of VHI setups.

In the present study, we want to investigate whether the illusion of embodying a virtual hand can be increased when haptic feedback is provided through wearable devices. Choi et al. investigated that regarding movement, a free movement task yields strongest illusions in different active movement conditions [8]. Furthermore, they reported that tactile stimulation had more significant influences than auditory stimulation on the VHI. The elicitation of the robotic hand illusion including vibrotactile feedback is reported in [29].

\subsection{Wearable Haptics}

In this work, the virtual hand illusion is performed without paintbrush stroking, but the free movement of the virtual hand is used to induce the illusion. Besides to the free movement, different types of haptic feedback could be used to strengthen the illusion since there are currently no scientific results implying which haptic feedback improves the illusion most. For this reason, the present paper tested whether haptic feedback can influence the strength of the virtual hand illusion. The focus of this paper is not only whether the illusion can be elicited or not but also whether the modality, i.e., force feedback and vibrotactile feedback, affects the strength of the illusion. In any case, it is necessary to exploit highly wearable interfaces to allow the hand of the user to freely move while being provided with a haptic feedback from the virtual environment.

Popular techniques to provide wearable haptic feedback usually consider moving platforms, pin-arrays, shearing belts and tactors, pneumatic jets, and balloon-based systems [12]. Frisoli et al. [30] presented a device composed of 
a parallel platform and a serial wrist; the parallel platform actuates a translation stage for positioning the plate with respect to the fingerpad, while the wrist is in charge of adjusting its orientation. A more wearable and improved design solution of the same system was later on presented in [31], [32]. Prattichizzo et al. [13] developed a wearable 3-DoF fingertip device consisting of two platforms: one located on the nail side of the finger, housing three DC motors, and the other one located in contact with the finger pulp. The two platforms are connected by three cables. The motors control the lengths of the cables to move the platform toward the user's fingertip and re-angle it to simulate contacts with arbitrarily oriented surfaces. A new version of this device featuring three articulated rigid legs connecting the two platforms has been presented in [33]. Minamizawa et al. [34] presented a wearable fingertip device consisting of two DC motors that move a belt in contact with the user's fingertip. When the motors spin in opposite directions, the belt presses into the user's fingertip, and when the motors spin in the same direction, the belt applies a tangential force to the skin. A similar device was also used in [35] for multi-finger manipulation of objects in VR. More recently, also Bianchi et al. [36] adopted a similar design for a fabric-based wearable display. Two DC motors move two pulleys attached to an elastic fabric band in contact with the fingertip, varying its stiffness. Moreover, a lifting mechanism can regulate the pressure exerted by the fabric band on the fingertip, enabling the device to render softness information, pressure normal to the fingertip skin, and slipping sensations. Leonardis et al. [37] developed a 3RSR wearable skin stretch device for the fingertip. It moves a rigid tactor in contact with the skin, providing skin stretch and making/breaking contact sensations. Girard et al. [38] developed a wearable fingertip device capable of rendering 2-DoF skin stretch stimuli. Two DC motors move a tactor in contact with the finger pulp, achieving a maximum displacement of $2 \mathrm{~mm}$ in both directions. Schorr and Okamura [39] presented a wearable device composed of a delta parallel mechanism, capable of making/breaking contact with the fingertip, as well as rendering shear and normal skin deformation stimuli. The device has three translational DoF, enabling normal, lateral, and longitudinal skin deformation. Feng et al. [40] presented

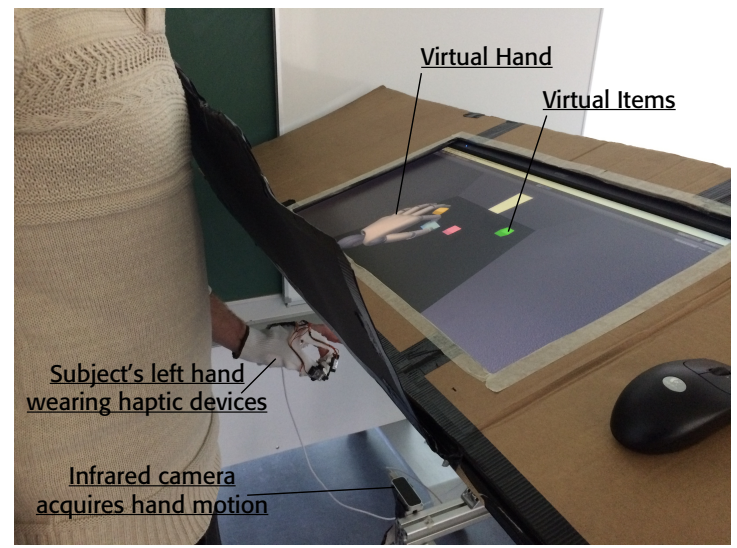

(a) a waterproof wearable fingertip display consisting of four miniature airbags. By inflating the airbags using a miniature speaker, the device can provide a wide range of vibrations as well as normal pressure to the fingertip.

\section{Method}

In the original rubber hand experiment, the rubber hand needs to lie at the table in an anatomically plausible position with the same position as the real hand within a distance of maximally $30 \mathrm{~cm}$ [15], [41]. Even though we use a free movement task, the virtual hand is located at an anatomically plausible position with an offset of $15 \mathrm{~cm}$ to the right. Previous works compared the laterality in the rubber hand illusion and reported, that the skin conductance responses, when watching the rubber hand being harmed, is stronger when the illusion was elicited on the left-hand [42]. Therefore, the left hand is focused to perform the virtual hand illusion in the experiment presented in the present paper.

Tsakiris and Haggard reported in 2005 that the rubber hand should look like one's own hand to properly induce the illusion. In the present study, a virtual prosthetic hand and wrist without a virtual arm were used (see Fig. 1) as the arm orientation was not tracked by the tracking system. The artificially prosthetic hand was chosen in contrast to a humanlike hand to avoid the uncanny valley [43].

Since one part of the illusion is the displacement of the perceived hand position towards the seen rubber hand, a common way to measure the strength of the illusion is to evaluate the perceived hand position [44]. This so-called proprioceptive drift effect is usually measured by asking the participants to close their eyes and point with the hand that is not part of the illusion the fingertip of the index finger of the hand that is part of the illusion. This method is mostly used when the illusion is induced passively only by stroking the hands without moving. We decided to consider proprioceptive drift even if, due to the problems with evaluating the drift in a active moving task as that proposed in this work, questionnaire are often used as the only mean to evaluate the strength of the illusion [8].

Finally, in the present study, the delay in the asynchronous condition has been set to $350 \mathrm{~ms}$ in addition to the

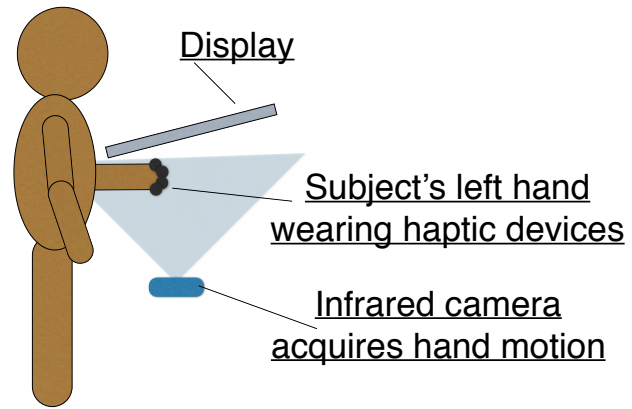

(b)

Fig. 1: Experimental setup. 
system-intrinsic delay to decrease the illusion significantly. Previous studies confirmed that the rubber hand illusion disappears when the artificial hand (e.g., a virtual hand) was touched asynchronously with the participants's hand [18], [45]. Shimada [45] examined the temporal discrepancy that is needed to decrease the illusion significantly. Their results showed that the temporal discrepancy of less than $300 \mathrm{~ms}$ between visual stimulation of the rubber hand and tactile stimulation of the changedparticipant'sparticipants's own hand is preferable to induce a strong illusion. In 2016, Ismail et al. transferred these results to robotic hand illusion (RoHI) that moved consistently with the participant's own hand. Their results showed that the participants felt a significantly greater effect of the RoHI with a visual feedback delay of less than about $200 \mathrm{~ms}$ compared with longer visual feedback delay [46]. Moreover, with a delay of 290-490$\mathrm{ms}$, the sense of agency occurred weaker and RoHI effect occurred for the ownership sense.

\subsection{Experimental setup}

For this experiment, participants were standing in front of a display while the participant's left hand that wore the glove with haptic devices was underneath the screen and thus hidden from their view as depicted in Fig. 1.

To hide the left hand, a cardboard cover was used. It covered $60 \mathrm{~cm}$ to the left, $20 \mathrm{~cm}$ to the top, $20 \mathrm{~cm}$ to the bottom and $50 \mathrm{~cm}$ to the right of the screen. Moreover, a movable cardboard cover lied on the participant's chest to hide the hand and also the arms' movement from the participant's view. On the right side of the cardboard, a computer mouse was placed to let the participants enter the perceived hand position and answer to the questionnaire proposed at the end of the experiment. An infrared camera (Leapmotion, USA) acquired hand motion and a virtual hand on the screen mimicked the hidden hands movement.The camera was used to acquire the hand motion similarly to the setup reported by Meli et al. in [47]. This controller was connected via USB and uses two monochromatic IR cameras and three infrared LEDs to track the fingertips' position in 3D. Weichert et al. analysed the accuracy with a robotic arm and achieved an accuracy of less than $2.5 \mathrm{~mm}$ $(S D=0.7)$ per axis [48]. A display (Iiyama - G-MASTER GB2788HS-B1, Japan) with $144 \mathrm{~Hz}$ was used to reduce the system-intrinsic delay and provide a fluently moving virtual hand. This screen was connected with a DisplayPort cable to a Gigabyte Radeon RX 460 WindForce 2G GPU (AMD, USA). The virtual environment was built with Unity Pro 3D game engine in version 4.6.6f2 that was given through the Unity License Grant Program (Unity, USA). The scene was originally part of "RobotStage" as part of the "Playground" application of Leap Motion that is open source available on GitHub. The screen and the camera were attached to an adjustable mount to fit the participant's height. During the experiment, the experimenter was hidden behind a wall to avoid any influence on the results. At the end of the experiment, participants were introduced by a text on the screen to call the experimenter to remove the haptic devices. In the following, we separately describe the constitutive elements of the experimental setup.

To explore the system-intrinsic delay, a high-speed camera with 240 frames per second was used to simultaneously record the real hand and the virtual hand. The experimenter moved his real hand in one direction, and the frames between the movement of the real hand and the movement of the virtual hand were counted. Results of this measurement showed that the system had a delay of $M=78.46 \mathrm{~ms}(S D=$ $6.67 \mathrm{~ms}$ ). According to [46], the additional delay was set to $350 \mathrm{~ms}$ on top of the system delay, and the procedure to determine the delay was repeated. Results of the asynchronous condition showed $M=430.0 \mathrm{~ms}(S D=18.26 \mathrm{~ms})$ of total delay which corresponds to $351.5 \mathrm{~ms}$ in addition to the system-intrinsic value. The standard deviation of the delay occurred due to different computational speed and refresh rates of the involved system components, i.e., LeapMotion, CPU, GPU, and display.

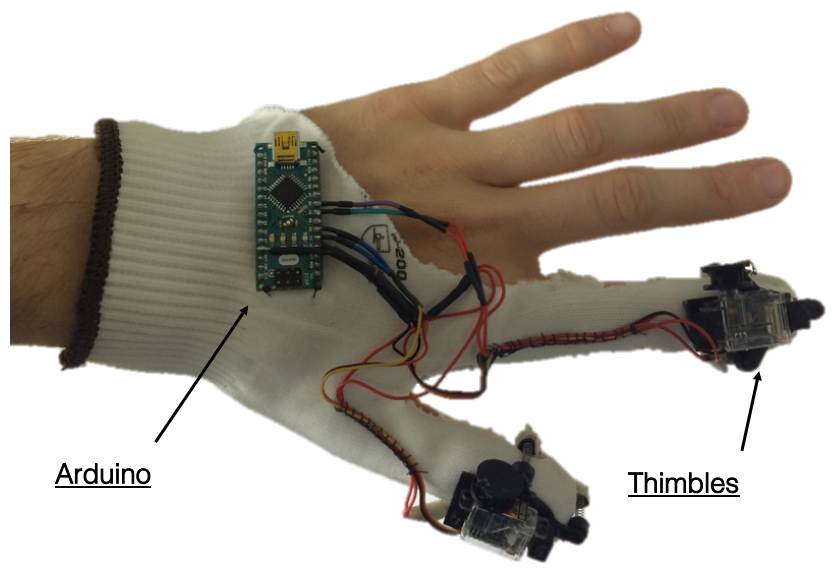

Fig. 2: The haptic glove used for the experiments. Two thimbles worn at the thumb and index provides both vibratory and normal force feedback.

For this experiment, two haptic devices and an Arduino Nano (Arduino, Italy) board were embedded on a glove to improve the wearability. The haptic device itself consists of two platforms connected by three strings and springs to keep the robes in place. The upper platform is passive and supports one servo motor with a pulley that is in charge of varying the length of the three robes to move the active platform. Since the finger is between those two platforms, force feedback is provided when the cable length is shortened by the servo, and thus the distance between the two platforms is reduced. All parts of the thimble were stiff and could not change their shape. This means that no edges or corners could be simulated as haptic feedback. The haptic device provides one linear degree of freedom (1-DoF) to control the contact force. A similar principle were presented in [47], [49], [50]. To provide vibrotactile feedback, a vibration motor was mounted on the active platform on the pulp-side of the finger. The motor used is a flat-type eccentric rotating mass vibrotactile motor (VPM2, Solarbotics, Canada). The vibrotactile motor was provided with an input voltage of $5 \mathrm{~V}$ when a contact occurred. The haptic system itself only weighs 10 grams including both platforms, the servo, the vibrotactile device and the cables that are connected to the Arduino. The glove with the whole haptic system was freely 
movable and thus did not cause any workspace restrictions. When the virtual hand was in contact with a virtual object, e.g., a virtual cube in the virtual environment, a signal was sent to the Arduino that either moved the servos or activates the vibrotactile devices according to the feedback mode selected. The whole experimental flowchart is shown in Fig. 3.

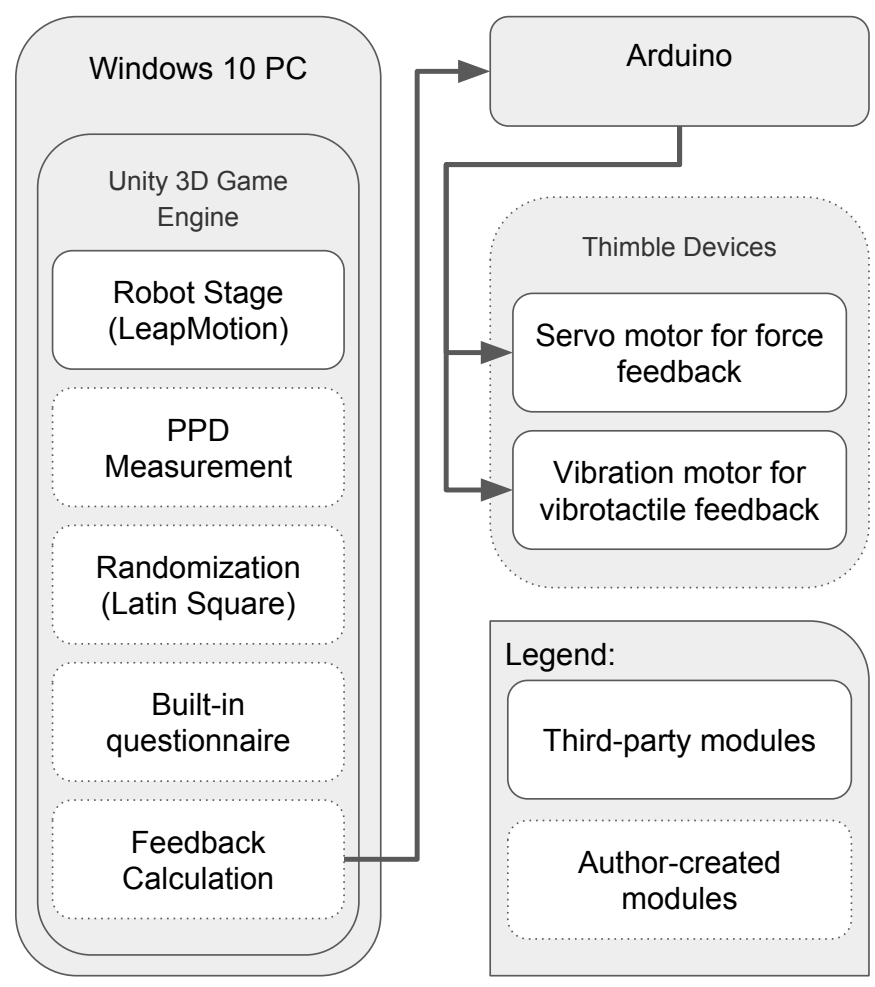

Fig. 3: Experimental flowchart. The experiment was running on a Windows 10 PC in the Unity 3D Game Engine. The proprioceptive drift (PPD) measurement, the randomization with Latin Square, and the embodiment questionnaire by Longo [18] were implemented in the experiment. The Arduino commanded the feedback through the haptic devices.

The modality of haptic feedback was treated as an independent variable. Touching a virtual object caused either vibrotactile feedback, force feedback or no feedback depending on the condition. The maximum stimulus was provided as soon as the object was touched, both for the vibrotactile feedback and the force feedback. This on/off feedback resulted to be more robust during the experiments with respect to a proportional feedback. To simplify the interaction between hand and object during grasping, the virtual cubes were programmed to stay between the fingers even when one finger lost contact to the object for a predefined time.

\subsection{Participants}

The optimal number of participants was calculated using the $\mathrm{G}^{*}$ Power analysis for repeated measures ANOVA for a within factor study. As limited prior knowledge about the effect size was available, an average effect size of $f=0.25$ was assumed according to previous studies in the literature. This analysis estimated a sample size of 28 participants, which was the goal of this study. We invited 32 participants in total and it turned out that all participants completed the whole study which means that no dropouts occurred and all data were usable for the final evaluation.

Thirty-two persons participated (11 female, 21 male; mean age $M=27.2, S D=7.2$ ) in this study. None of the participants reported weaknesses in their visual or haptic perception abilities. Three of them were left-hand dominated, 29 right-hand dominated. To avoid any external cues, participants wore passive noise canceling headphones and the laboratory was not used by other people during the experiments.

All participants were students or employees of Technische Universität Darmstadt and participated voluntarily. At the beginning of the experiment, all participants gave informed consent where the potential risks during the experiment were mentioned. All participants were naive to the specific design of the study focusing on the influence of different haptic feedback modalities on bodily illusions. In the questionnaire, we surveyed whether the participants knew the concept of the rubber hand illusion in general and found that 15 were aware. Due to the within-subject design, the results represent the relative differences for each individual subject such as the differences in embodiment occurred due to different haptics or delays.

The study was under the ethical guidelines of the European Project WEARHAP coordinated by the University of Siena.

\subsection{Task}

Participants were asked to put a virtual cube on a virtual moving target. As displayed in Fig. 1 and Fig. 4 , four different colored cubes spawned at the beginning of the experiment. Participants were asked to put each virtual cube on one of the randomly moving virtual objects which had the shape of a robot without a head. The robot started dancing as soon as a colored cube was placed as a head on top of the moving robot.

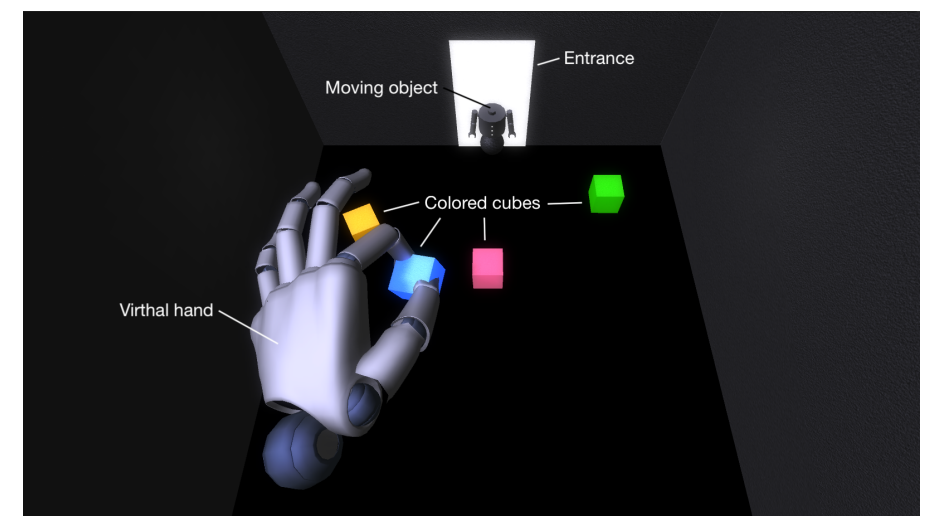

Fig. 4: Experimental scene. The moving virtual object (a robot) is entering the scene through the white door and starts to move randomly around in the virtual room. Participants are asked to place one colored cube on the robot

The first robot entered the scene through the white entrance (see Fig. 4) after eight seconds. As soon as one of a colored cubes was attached to the head of a robot, another 
robot entered the scene to a maximum of four robots to have one robot for each colored cube. When all four colored cubes were on top of a robot, a disco light appeared to show the participants that they completed the task. After 5 seconds the disco light and all moving objects disappeared, and the scene restarted with spawning the colored cubes. To keep the task challenging for the participants, all moving targets disappeared after 30 seconds to reset the scene. This time window challenged the participants to put all four cubes during the 30 seconds.

\subsection{Measures}

Three dependent variables were measured for every single condition: subjective embodiment, proprioceptive drift, and task performance. These data were collected during and after each condition to have autonomous measurements to determine differences between conditions.

\subsubsection{Subjective embodiment}

Longo et al. developed a questionnaire scale to measure embodiment with ten items [18]. This questionnaire is used to psychometrically assess the embodiment of the external hand (originally of the rubber hand) and consists of three subscales: ownership, location, and agency. These items explain up to 75 percent variance of the strength of the illusion.

The questionnaire was embedded in the virtual scene where participants were asked to use a mouse with their right hands as this hand was not wearing any haptic devices. To prevent memory effects, a visual analog scale ranging from "strongly disagree" to "strongly agree" with a length of $93 \mathrm{~mm}$ was used in the present study, instead of the 7 point likert scale ranging from -3 to 3 that was used by Longo et al. Participants moved a slider between -3 and 3 that was displayed as "strongly disagree" and "strongly agree". The slider started in the middle of the scale for every question and had to be moved at least a little. Otherwise, an error message asked the participant to move the slider to proceed whereas moving back to the middle of the slider was also possible.

All items were provided after the proprioceptive drift measurement and in a computer-controlled random order. Since data was collected in Germany, the original items were translated into German and adapted from rubber hand to virtual hand. Furthermore, question number 8 , that is part of the subscale location, was changed from "It seemed like the touch I felt was caused by the paintbrush touching the rubber hand" to "During the block it seemed like the touch I felt was caused by touching the virtual cube". In table 1, all items from the questionnaire by Longo are displayed in their modified English version and the translated version in German as used in the study.

\subsubsection{Proprioceptive drift}

The proprioceptive drift (PPD) is an objective measurement for the embodiment of an external limb without a survey. PPD describes the tendency to ascribe the location of one's own arm to that of the virtual hand after a successfully evoked illusion [51].
TABLE 1: Items from the Embodiment questionnaire by Longo in its English version (left) and its translated version (right) that is used in this study.

\begin{tabular}{lll}
\hline English statement & $\begin{array}{l}\text { Translated statement (Ger- } \\
\text { man) }\end{array}$ \\
During the block... & Während des letzten \\
& Blocks... & \\
\hline
\end{tabular}

Subscale ownership
... it seemed like I was
looking directly at my own
hand, rather than at a vir-
tual hand.
$\ldots$ it seemed like the virtual
hand began to resemble my
real hand.
... it seemed like the virtual
hand belonged to me.

... it seemed like the virtual hand was my hand.

... hatte ich den Eindruck, direkt meine eigene Hand anzuschauen, nicht eine virtuelle Hand..

... schien es, als ob die virtuelle Hand meiner realen Hand ähnlicher wurde.

... schien es, als ob die virtuelle Hand $\mathrm{zu}$ mir gehören würde.

... schien es, als ob die virtuelle Hand meine eigene Hand war.

... it seemed like the virtual ... schien es, als ob die hand was part of my body. virtuelle Hand ein Teil meines Körpers war.

Subscale Location

... it seemed like my hand was in the location where the virtual hand was.

... schien es, als ob meine eigene Hand in der Position der virtuellen Hand war.

... it seemed like the virtual hand was in the location where my hand was.

... schien es, als ob die künstliche Hand in der Position meiner eigenen Hand war.

... it seemed like the touch I felt was caused by touching the virtual cube

... hatte ich den Eindruck, dass die Berührung, die ich fühlte, durch das Berühren des virtuellen Würfels verursacht wurde.

\section{Subscale Agency}

... it seemed like I could have moved the virtual hand if I had wanted.

... it seemed like I was in control of the virtual hand.

... hatte ich den Eindruck, dass ich die virtuelle Hand bewegen könnte, wenn ich gewollt hätte.

... hatte ich den Eindruck, die Kontrolle über die virtuelle Hand zu haben.

Researchers observed that the estimated position is shifting more toward the artificial hand (e.g., rubber hand, virtual hand, robotic hand) when the strength of the illusion increases [14], [18] [15], [52].

The proprioceptive drift was measured at the end of each condition. Therefore, the screen turned black right after the interaction time of three minutes was completed. 
Participants were asked to use their right hand to click the mouse cursor to the perceived position of their left index finger while they were introduced to not move their left hand during this measurement. The left hand was still hidden under the screen and the cardboard cover and thus hidden from the participants view. The proprioceptive drift was measured once after for each condition.

\subsubsection{Task performance}

Participants were asked to put the cubes on the moving target. The number of successfully placed cubes on a moving target is the third dependent variable.

\subsection{Procedure}

Before the experiment started, participants were asked to read and sign the informed consent. After informed consent was given, participants were positioned in front of the experimental setup to adjust its height to fit the height of the subject. Afterward, participants put on the glove with the haptic devices (see Fig. 2), which were then individually calibrated to fit the participant's finger size. Participants received oral information on the LeapMotion camera functioning. The virtual task was designed so to naturally require a pinch grasp executed with thumb and index fingers where haptic interfaces were placed. Furthermore, this experimental task suits LeapMotion tracking since it minimizes possible occlusions due to the specific hand orientation. When the participant felt comfortable with the glove and the height of the setup, the experimenter started the actual experiment and went behind a wall to avoid experimenter-caused biases during the experiment. All participants performed all six conditions, with a duration of three minutes each, in a randomized order using the Latin square design. As soon as the experiment was started, no further actions of the experimenter were needed as the entire workflow was automatically controlled by the computer. Therefore, one of the six conditions was randomly chosen by the computer using the Latin square design. The condition started as described in 3.3 until the condition time of three minutes was reached. Afterwards, the proprioceptive drift was measured as described in 3.4.2 followed by the questionnaire 3.4.1 which were also provided at the virtual screen as part of the virtual environment. When the questionnaire was completed, the next condition of the Latin square design started to follow the same procedure as the first condition. This procedure was completed for all six conditions. The only difference between conditions was the provided feedback to the participant's left hand and whether the hand was displayed synchronously or asynchronously.

To determine differences in embodiment, proprioceptive drift, and task performance between conditions, two-way repeated measures ANOVA will be used. Before calculating the ANOVA, all relevant assumptions such as normal distribution and sphericity will be tested. When one or more assumption is validated, an equivalent test will be conducted.

\section{Results}

Since all data showed no significant deviation from normal distribution as displayed in Table 2, a two-way repeated measures ANOVA was used to determine relevant differences for all three dependent variables, i.e., subjective embodiment, proprioceptive drift, and task performance. In case of sphericity, the Greenhouse-Geisser correction was used to calculate the ANOVA.

TABLE 2: Test of Normality (Komogorov-Smirnov-Test). Significant results suggest a deviation from normality.

\begin{tabular}{lcc}
\hline Dependent Variable & Synchronously & Asynchronously \\
\hline PPD & $p=.993$ & $p=.322$ \\
Embodiment & $p=.817$ & $p=.631$ \\
Performance & $p=.729$ & $p=.735$ \\
\hline
\end{tabular}

Paired $t$-Tests were calculated one-sided for each hypothesis. The significance level was adjusted by the Bonferroni alpha-error correction for each dependent variable separately and is displayed under each diagram.

To verify the results of the classical hypothesis testing, the Bayes-Factor was calculated. With this method, both the null hypothesis and the alternative hypothesis can be supported in contrast to the classical hypothesis testing where only evidence against the null hypothesis is possible. For the interpretation of the Bayes-Factor, the scale of Kass and Raftery is used [53].

\subsection{Subjective embodiment}

Embodiment represents the mean questionnaire rating of all 10 Items for each participant and condition. Fig. 5 displays the means and standard deviations of Embodiment questionnaire (see table 1 ) ratings for all synchronous conditions.

To explore whether the conditions differ from each other or not, a two-way repeated measure ANOVA with the factors "Conditions" and "Delay" was calculated. This ANOVA yielded a significant main effect of conditions and Delay as displayed in Table 3. Since the Machly's test of sphericity indicates that the assumption of sphericity is violated, the Greenhouse-Geisser sphericity correction was used to calculate this ANOVA.

\begin{tabular}{lll}
\hline Condition & $F$-Value & $p$-Value \\
\hline Feedback & $F=9.771$ & $p<.001^{* *}$ \\
Delay & $F=10.4405$ & $p=.003^{*}$ \\
Feedback* Delay & $F=0.311$ & $p=.734$ \\
\hline No Feedback & Force Feedback & $p=.002^{*}, d=.808$ \\
No Feedback & Vibrotactile & $p=.006^{*}, d=.579$ \\
& Feedback & \\
Force Feedback & Vibrotactile & $p=.102, d=.287$ \\
& Feedback & \\
\hline
\end{tabular}

TABLE 3: Upper part: Emobdiment ANOVA output.

Lower Part: Post-Hoc comparison for synchronous conditions with one-tailed paired $t$-Test and Effect size by Cohen (Cohens $d$ ).

Post-Hoc comparison for synchronous conditions, calculated with a one-tailed paired $t$-Test and Bonferroni $\alpha$ error correction to $\alpha$-error $.05 / 3=.017$, indicates significant 
results between "No Feedback" and "Force Feedback" ( $p$ $=.002)$, "No Feedback" and "Vibration Feedback" ( $p=.006)$ and no significant result between "Force Feedback and "Vibration Feedback" ( $p=.102)$.

The Bayes-Factor supports the significant results of the ANOVA in both main effects. Feedback leads a value of $B F_{10}=390.8$, which gives very strong evidence that variations of feedback yield differences of embodiment concerning the interpretation of Kass and Raftery. The Bayes-Factor of $B F_{10}=56.5$ for delay shows strong evidence that different delays also influence the embodiment results. The combination of feedback and delay also results again in very strong evidence that the combination between different feedback modalities and delay results in different embodiment with a Bayes Factor of $B F_{10}=42288.3$.

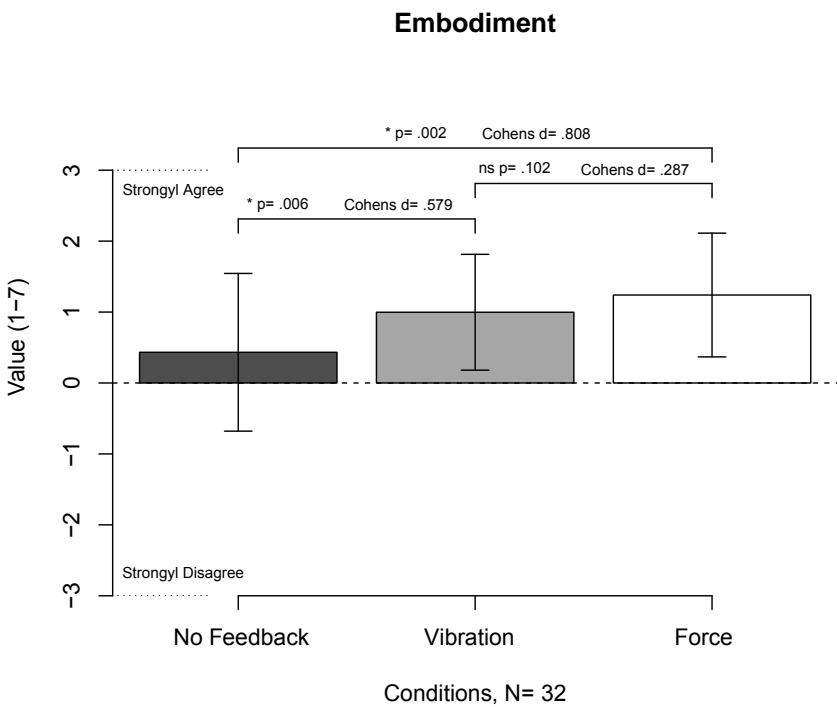

Fig. 5: Means and standard deviation of the whole Embodiment questionnaire by Longo [18]. Bonferroni correction $\alpha=.05 / 3=.017$. These $p$-Values of a paired sample $t$-Test support the results of the ANOVA that there are significant differences in the embodiment between synchronous conditions.

To examine, where exactly the differences between conditions are, three additional ANOVAs have been calculated for the subscales Ownership, Location, and Agency. The results of this analysis are displayed in table 4 .

\begin{tabular}{llll}
\hline Variable & Feedback & Delay & Feedback * Delay \\
\hline Embodiment & $p<.001^{* *}$ & $p=.003^{*}$ & $p=.734$ \\
Agency & $p=.246$ & $p=.008^{*}$ & $p=.828$ \\
Ownership & $p=.141$ & $p<.001^{* *}$ & $p=.520$ \\
Location & $p<.001^{* *}$ & $p=.009^{*}$ & $p=.481$ \\
\hline
\end{tabular}

TABLE 4: ANOVA for each subscale of Embodiment questionnaire.

Post-Hoc analysis were only calculated for the subscale Location as the results for the other subscales were not significant regarding the main effect feedback. The onetailed paired $t$-Test showed significant differences between "No Feedback" and "Force Feedback" $\left(p<.001^{* *}\right)$ as well as between "No Feedback" and "Vibrotactile Feedback" $\left(p<.001^{* *}\right)$. Between "Vibrotactile Feedback" and "Force Feedback", however, no significant difference $(p=.093)$ was observed.

\section{During the block it seemed like} the virtual hand was my hand.

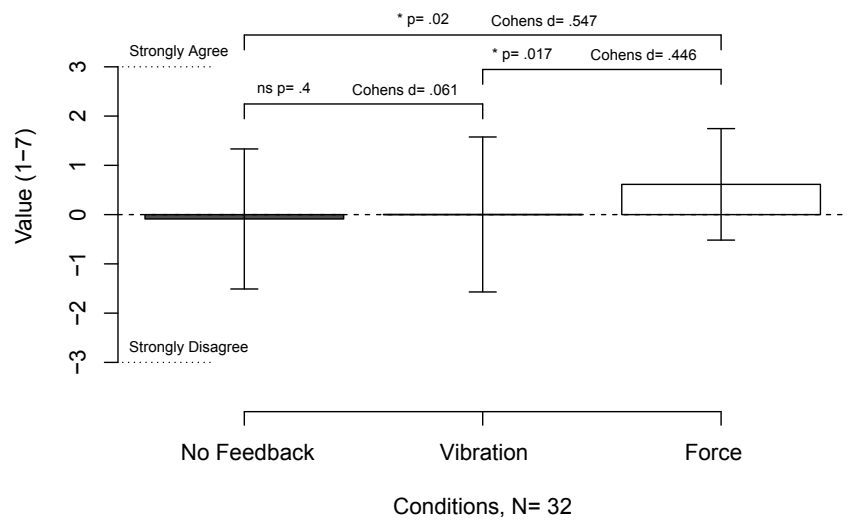

Fig. 6: Means and standard deviation of the questione "During the block it seemed like the virtual hand was my hand" as part of the Embodiment questionnaire by Longo [18].

The result of one particular question of the embodiment questionnaire indicates that participants have a significantly stronger sensation that the virtual hand was their own hand during the condition where force feedback was provided as depicted in Fig. 6.

For this specific item, the mean questionnaire value after the synchronous condition with force feedback $(M=.614 S D$ $=1.132)$ was significantly higher $\left(p=.020^{*}\right)$ than the mean questionnaire value after the condition without feedback $(M$ $=-.088 S D=1.421)$ and also significantly higher $\left(p=.017^{*}\right)$ than the mean questionnaire value after the condition with vibrotactile feedback $(M=-.003 S D=1.573)$.

These results are supported by a Bayesian Repeated Measures ANOVA with a Bayes Factor of $\mathrm{BF}_{10}=19.0$, which is a positive to strong effect regarding the interpretation by Kass and Raftery.

\subsection{Proprioceptive drift}

To investigate the differences in the proprioceptive drift, another two-way repeated measure ANOVA was calculated. Results indicate that the proprioceptive drift was neither affected by the main effect of condition nor by the delay as displayed in table 5. Post-Hoc comparison with onetailed paired $t$-Test strengthen these results (see Fig. 7) since the standard deviation is too large in all synchronous conditions.

The Bayes-Factor shows similar results in both main effects (Feedback $\mathrm{BF}_{10}=0.238$ and Delay $\left.\mathrm{BF}_{10}=.354\right)$ and Interaction $\left(\mathrm{BF}_{10}=.082\right)$. 


\begin{tabular}{lll}
\hline Condition & $F$-Value & $p$-Value \\
\hline Feedback & $F=11.416$ & $p=.236$ \\
Delay & $F=11.549$ & $p=.136$ \\
Feedback * Delay & $F=3.137$ & $p=.617$ \\
\hline
\end{tabular}

TABLE 5: PPD ANOVA output.

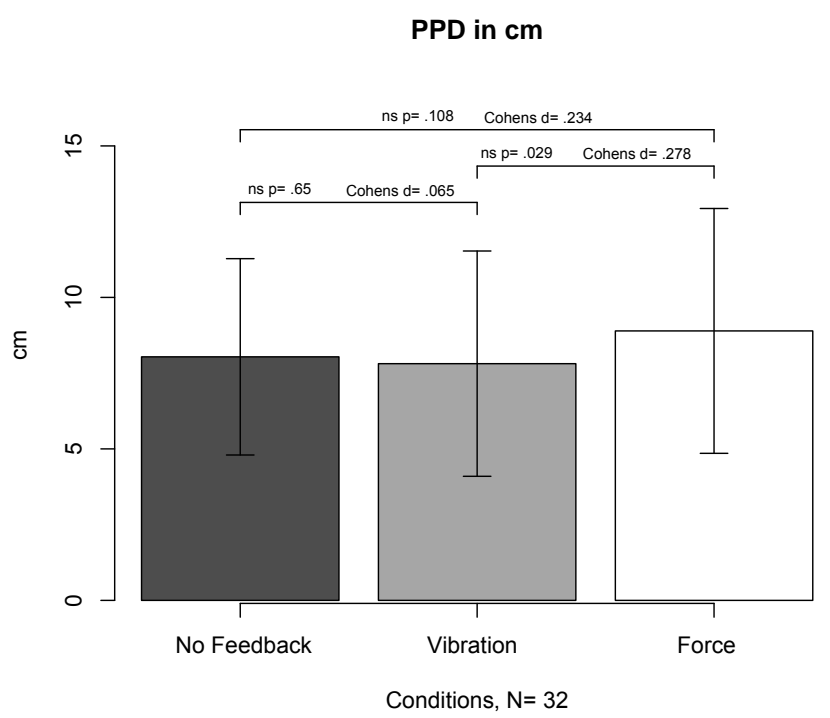

Fig. 7: Means and standard deviation of PPD. Bonferroni correction $\alpha=.05 / 3=.017$. These $p$-Values of a paired sample $t$-Test support the results of the ANOVA that there are no significant differences in the PPD between all three synchronous conditions.

\subsection{Task performance}

For the third dependent variable, another repeated measures ANOVA was calculated. Results indicate no significant main effect of feedback $(p<.963)$ As expected, participants were able to put significant more cubes on a moving target $\left(p<.001^{* *}\right)$ when no additional delay was provided. The Bayesian Repeated Measures ANOVA shows similar results with a Bayes Factor of $\mathrm{BF}_{10}=.057$ for Feedback and $\mathrm{BF}_{10}=$ 1326.7 for Delay which is a very strong effect regarding the interpretation of Kass and Raftery.

\begin{tabular}{lll}
\hline Condition & $F$-Value & $p$-Value \\
\hline Feedback & $F=.038$ & $p=.963$ \\
Delay & $F=23.68$ & $p<.001^{* *}$ \\
Feedback * Delay & $F=.026$ & $p=.975$ \\
\hline
\end{tabular}

TABLE 6: Cubes put on the moving target - ANOVA output.

\section{Discussion}

This study investigates, whether and how haptic feedback influences the body experience of humans in virtual environments. Specific focus is set on comparing different modalities, i.e., vibrotactile and force feedback. We report

\begin{tabular}{llll}
\hline Condition & Location & Agency & Ownership \\
\hline Feedback & $p<.001^{* *}$ & $p=.246$ & $p=.141$ \\
Delay & $p=.009^{*}$ & $p=.008^{*}$ & $p<.001^{* *}$ \\
Feedback * Delay & $p=.481$ & $p=.828$ & $p=.520$ \\
\hline
\end{tabular}

TABLE 7: Three different ANOVA output of Location, Agency and Ownership.

\begin{tabular}{llll}
\hline Variable & Feedback & Delay & Feedback ${ }^{*}$ Delay \\
\hline PPD & $p=.236$ & $p=.136$ & $p=.617$ \\
Embodiment & $p<.001^{* *}$ & $p=.003^{*}$ & $p=.734$ \\
Agency & $p=.246$ & $p=.008^{*}$ & $p=.828$ \\
Ownership & $p=.141$ & $p<.001^{* *}$ & $p=.520$ \\
Location & $p<.001^{* *}$ & $p=.009^{*}$ & $p=.481$ \\
Performance & $p=.963$ & $p<.001^{* *}$ & $p=.975$ \\
\hline
\end{tabular}

TABLE 8: All ANOVA $p$-Values.

results for differences in subjective embodiment of a virtual hand between haptic modalities. These results may be used by designers and developers to build realistic and qualitative feedback that meet the psychological requirements for human-centered applications [54].

Haptic perception can be considered as a subjective sensation that can be evaluated by subjective methods such as questionnaires. In the present study, subjective embodiment of a virtual hand was measured to analyze the quality of different haptic modalities. The experimental results imply that haptic feedback devices can alter human body experience and foster the subjective embodiment of non-corporal limb avatars. Even though the experience of bodily illusions can vary largely across individuals [55], the exertion of tactile feedback yielded significantly increased subjective embodiment. In particular, the embodiment questionnaire by Longo [18] indicates that certain experiences of ownership were additionally increased if force feedback was provided. This is underlined by the replies to the item "During the block it seemed like the virtual hand was my hand": force feedback led to a higher response with respect to all other feedback modalities. Participants have a significantly stronger sensation that the virtual hand was their hand during the condition where force feedback was provided. This result might mean that the skin deformation induced by the moving platform is perceived as more realistic with respect to a vibration. However, all other questionnaire items did not show significant differences between force feedback and vibrotactile feedback results. These questionnaire results show that feedback, and in particular force feedback, enhances subjective embodiment and should be considered by haptic designers such as developers for teleoperation and VR applications.

Furthermore, the proprioceptive drift as an objective measurement shows an increased shift in the perceived proprioception towards the location of the virtual hand that was close to be significant between haptic feedback and no feedback as well as between force feedback and vibrotactile feedback. Conforming with previous observa- 
tions, introducing a delay of $430 \mathrm{~ms}$ significantly affected embodiment and task performance. After the experiments, participants were asked whether they noticed the delay in three of out of six conditions and only one participant reported that he noticed the difference, which underlines the applicability of the overall delay. The lack of significant differences in the proprioceptive drift between conditions may be due to vision predominance over proprioception and kinesthesia. Vision appears to be an important aspect of multisensory integration and it was reported to be dominant in previous research [14], [56]. Although other studies also argue against vision dominance [57], this might explain rather small differences of proprioceptive drift observed in the present study. An additional explanation could be found considering the definition of proprioception by [58] where proprioception is defined as the unconscious perception of movement and spatial orientation". As we provided an active movement task in the present study, the proprioception continuously updated in all conditions and led decreased the impact of the seen virtual hand. Additionally, proprioception is not linked to haptic feedback, which may explains the lack of differences between conditions. Generally, the rubber hand illusion is due to a crossmodal blending of the visual and proprioceptive experience of the hand position [14]. In this experiment, the proprioceptive experience continuously varied since the participants needed to move their hand to perform the task. This might have influenced the illusion in addition to the variation of feedback modality, which might be the reason why the proprioceptive drift exists in all conditions but does not differ between them significantly. Rohde et al. reported that subjective ratings and proprioceptive drift are dissociated [44]. They conclude that different mechanisms of multisensory integration are responsible for proprioceptive drift and the feeling of ownership. These findings fit the results in the present study since the difference between conditions in the embodiment are not observed in the proprioceptive drift. In future studies, the virtual hand and the real hand might be placed congruently instead of displacing the virtual hand with an offset of $15 \mathrm{~cm}$. This offset is inevitable for the original rubber hand illusion, but not for the virtual hand illusion. Yet, by placing both hands at the same location, the proprioceptive drift could no longer be measured. Zhang et al. investigated the influence of ownership and distance between the real hand and the virtual hand. They reported that the experience of ownership increases with decreasing distance [59] and that the impact of synchrony was stronger for short distances. This underlines that limb congruity where the virtual hand is provided at the same place as the real hand should strengthen the subjective embodiment.

\section{Conclusion}

This within-subject study provides a preliminary comparison of different feedback modalities in the virtual hand illusion. Behavioral questionnaire measurements show a significantly higher sensation of embodying the virtual hand when vibrotactile or force feedback is provided. Moreover, force feedback yielded higher scores in one ownershiprelated item and delaying the virtual hand by $350 \mathrm{~ms}$ decreased the subjective experience of embodiment significantly. Christ et al. [51] state that the "virtual hand illusion appeared to be less intense than when it was supported by visual, haptic stimuli as measured by physiological and behavioral indications." These findings fit the results of the present study since the behavioral measurement, i.e., the embodiment questionnaire, showed an increased intensity of the illusion while the proprioceptive drift did not.

These findings can provide guidelines for the design of haptic devices and the creation of virtual environments since both, the haptic feedback modality and the delay of the system, are important to evoke the experience that the virtual hand is the participants own hand.

\section{REFERENCES}

[1] W. Sadowski and K. Stanney, "Presence in virtual environments." 2002.

[2] F. Biocca, "The cyborg's dilemma: Progressive embodiment in virtual environments," Journal of Computer-Mediated Communication, vol. 3, no. 2, pp. 0-0, 1997.

[3] M. R. Costa, S. Y. Kim, and F. Biocca, "Embodiment and embodied cognition," in International Conference on Virtual, Augmented and Mixed Reality. Springer, 2013, pp. 333-342.

[4] M. Slater, D. Pérez Marcos, H. Ehrsson, and M. V. Sanchez-Vives, "Towards a digital body: the virtual arm illusion," Frontiers in human neuroscience, vol. 2, p. 6, 2008.

[5] C. Basdogan, C.-H. Ho, M. A. Srinivasan, and M. Slater, "An experimental study on the role of touch in shared virtual environments," ACM Transactions on Computer-Human Interaction (TOCHI), vol. 7, no. 4, pp. 443-460, 2000.

[6] R. Stone, "Haptic feedback: A brief history from telepresence to virtual reality," Haptic Human-Computer Interaction, pp. 1-16, 2001.

[7] E.-L. Sallnäs, K. Rassmus-Gröhn, and C. Sjöström, "Supporting presence in collaborative environments by haptic force feedback," ACM Transactions on Computer-Human Interaction (TOCHI), vol. 7, no. 4, pp. 461-476, 2000.

[8] W. Choi, L. Li, S. Satoh, and K. Hachimura, "Multisensory Integration in the Virtual Hand Illusion with Active Movement," BioMed Research International, vol. 2016, p. e8163098, 2016. [Online]. Available: https://www.hindawi.com/journals/bmri/ 2016/8163098/abs/

[9] M. Hara, H. Nabae, A. Yamamoto, and T. Higuchi, "A novel rubber hand illusion paradigm allowing active self-touch with variable force feedback controlled by a haptic device," IEEE Transactions on Human-Machine Systems, vol. 46(1), pp. 78-87, 2016.

[10] M. V. Sanchez-Vives and M. Slater, "From presence to consciousness through virtual reality," Nature Reviews Neuroscience, vol. 6, no. 4, pp. 332-339, 2005.

[11] M. A. Padilla-Castañeda, A. Frisoli, S. Pabon, and M. Bergamasco, "The modulation of ownership and agency in the virtual hand illusion under visuotactile and visuomotor sensory feedback," Presence: Teleoperators and Virtual Environments, vol. 23, no. 2, pp. 209-225, 2014.

[12] C. Pacchierotti, S. Sinclair, M. Solazzi, A. Frisoli, V. Hayward, and D. Prattichizzo, "Wearable haptic systems for the fingertip and the hand: taxonomy, review, and perspectives," IEEE Transactions on Haptics, 2017

[13] D. Prattichizzo, F. Chinello, C. Pacchierotti, and M. Malvezzi, "Towards wearability in fingertip haptics: a 3-dof wearable device for cutaneous force feedback," IEEE Transactions on Haptics, vol. 6 , no. 4, pp. 506-516, 2013.

[14] M. Botvinick, J. Cohen, and others, "Rubber hands' feel'touch that eyes see," Nature, vol. 391, no. 6669, pp. 756-756, 1998.

[15] M. Tsakiris and P. Haggard, "The rubber hand illusion revisited: visuotactile integration and self-attribution," Journal of Experimental Psychology. Human Perception and Performance, vol. 31, no. 1, pp. 80-91, Feb. 2005.

[16] L. Raz, P. L. Weiss, and M. Reiner, "The virtual hand illusion and body ownership," in International Conference on Human Haptic Sensing and Touch Enabled Computer Applications. Springer, 2008, pp. 367-372. 
[17] S. Arzy, L. S. Overney, T. Landis, and O. Blanke, "Neural mechanisms of embodiment: asomatognosia due to premotor cortex damage," Archives of Neurology, vol. 63, no. 7, pp. 1022-1025, Jul. 2006.

[18] M. R. Longo, F. Schüür, M. P. Kammers, M. Tsakiris, and P. Haggard, "What is embodiment? A psychometric approach," Cognition, vol. 107, no. 3, pp. 978-998, Jun. 2008.

[19] P. Beckerle, C. Castellini, and B. Lenggenhager, "Robotic interfaces for cognitive psychology and consciousness research: a research roadmap," Wiley Interdisciplinary Reviews: Cognitive Science, in press.

[20] F. Short and R. Ward, "Virtual limbs and body space: Critical features for the distinction between body space and near-body space." Journal of Experimental Psychology: Human Perception and Performance, vol. 35, no. 4, p. 1092, 2009.

[21] E. A. Caspar, A. De Beir, P. A. Magalhaes De Saldanha Da Gama, F. Yernaux, A. Cleeremans, and B. Vanderborght, "New frontiers in the rubber hand experiment: when a robotic hand becomes ones own," Behavior Research Methods, vol. 47, no. 3, pp. 744-755, Sep. 2015.

[22] D. Romano, E. Caffa, A. Hernandez-Arieta, P. Brugger, and A. Maravita, "The robot hand illusion: Inducing proprioceptive drift through visuo-motor congruency," Neuropsychologia, vol. 70, pp. 414-420, Apr. 2015.

[23] K. Ma, D. P. Lippelt, and B. Hommel, "Creating Virtual-hand and Virtual-face Illusions to Investigate Self-representation," JoVE (Journal of Visualized Experiments), no. 121, pp. e54784-e54784, Mar. 2017. [Online]. Available: https://www.jove.com/video/54784/ creating-virtual-hand-virtual-face-illusions-to-investigate-self

[24] M. Slater, D. Prez Marcos, H. Ehrsson, and M. V. Sanchez-Vives, "Inducing illusory ownership of a virtual body," Frontiers in neuroscience, vol. 3, p. 29, 2009.

[25] N. David, "New frontiers in the neuroscience of the sense of agency," Frontiers in Human Neuroscience, vol. 6, p. 161, 2012. [Online]. Available: https://www.frontiersin.org/article/ 10.3389/fnhum.2012.00161

[26] K. Ma and B. Hommel, "The virtual-hand illusion: effects of impact and threat on perceived ownership and affective resonance," Frontiers in Psychology, vol. 4, 2013.

[27] W. A. IJsselsteijn, Y. A. W. de Kort, and A. Haans, "Is This My Hand I See Before Me? The Rubber Hand Illusion in Reality, Virtual Reality, and Mixed Reality," Presence: Teleoperators and Virtual Environments, vol. 15, no. 4, pp. 455-464, Aug. 2006. [Online]. Available: http://dx.doi.org/10.1162/pres.15.4.455

[28] P. Beckerle, A. De Beir, T. Schürmann, and E. A. Caspar, "Human body schema exploration: Analyzing design requirements of Robotic Hand and Leg Illusions," in Robot and Human Interactive Communication (RO-MAN), 2016 25th IEEE International Symposium on. IEEE, 2016, pp. 763-768.

[29] A. M. Abrams and P. Beckerle, "A pilot study: Advances in robotic hand illusion and its subjective experience," in HRI Pioneers 2018 (ACM/IEEE International Conference on Human-Robot Interaction), 2018, pp. 289-290.

[30] A. Frisoli, M. Solazzi, F. Salsedo, and M. Bergamasco, "A fingertip haptic display for improving curvature discrimination," Presence: Teleoperators and Virtual Environments, vol. 17, no. 6, pp. 550-561, 2008.

[31] M. Solazzi, A. Frisoli, and M. Bergamasco, "Design of a novel finger haptic interface for contact and orientation display," in Proc. IEEE Haptics Symposium, 2010, pp. 129-132.

[32] M. Gabardi, M. Solazzi, D. Leonardis, and A. Frisoli, "A new wearable fingertip haptic interface for the rendering of virtual shapes and surface features," in Proc. IEEE Haptics Symposium, 2016, pp. 140-146.

[33] F. Chinello, M. Malvezzi, C. Pacchierotti, and D. Prattichizzo, "Design and development of a 3RRS wearable fingertip cutaneous device," in Proc. IEEE/ASME Int. Conf. Advanced Intelligent Mechatronics, 2015, pp. 293-298.

[34] K. Minamizawa, S. Fukamachi, H. Kajimoto, N. Kawakami, and S. Tachi, "Gravity grabber: wearable haptic display to present virtual mass sensation," in Proc. ACM SIGGRAPH emerging tech., 2007, p. 8.

[35] C. Pacchierotti, G. Salvietti, I. Hussain, L. Meli, and D. Prattichizzo, "The hRing: a wearable haptic device to avoid occlusions in hand tracking," in Proc. IEEE Haptics Symposium, 2016.
[36] S. Fani, S. Ciotti, E. Battaglia, A. Moscatelli, and M. Bianchi, "Wfyd: a wearable fabric-based display for haptic multi-cue delivery and tactile augmented reality," IEEE Transactions on Haptics, 2017.

[37] D. Leonardis, M. Solazzi, I. Bortone, and A. Frisoli, "A 3-RSR haptic wearable device for rendering fingertip contact forces," IEEE Trans. Haptics, 2016.

[38] A. Girard, M. Marchal, F. Gosselin, A. Chabrier, F. Louveau, and A. Lécuyer, "Haptip: Displaying haptic shear forces at the fingertips for multi-finger interaction in virtual environments," Frontiers in ICT, vol. 3, p. 6, 2016.

[39] S. B. Schorr and A. M. Okamura, "Fingertip tactile devices for virtual object manipulation and exploration," in Proc. CHI Conf. Human Factors in Computing Systems, 2017, pp. 3115-3119.

[40] Y.-L. Feng, C. L. Fernando, J. Rod, and K. Minamizawa, "Submerged haptics: a 3-dof fingertip haptic display using miniature 3d printed airbags," in Proc. ACM SIGGRAPH - Emerging Tech., 2017, p. 22.

[41] D. M. Lloyd, "Spatial limits on referred touch to an alien limb may reflect boundaries of visuo-tactile peripersonal space surrounding the hand," Brain and Cognition, vol. 64, no. 1, pp. 104-109, Jun. 2007.

[42] S. Ocklenburg, N. Rther, J. Peterburs, M. Pinnow, and O. Gntrkn, "Laterality in the rubber hand illusion," Laterality, vol. 16, no. 2, pp. 174-187, Mar. 2011.

[43] M. Mori, K. MacDorman, and N. Kageki, "The Uncanny Valley [From the Field]," IEEE Robotics \& Automation Magazine, vol. 19 pp. 98-100, Jun. 2012.

[44] M. Rohde, M. Di Luca, and M. O. Ernst, "The Rubber Hand Illusion: feeling of ownership and proprioceptive drift do not go hand in hand," PloS One, vol. 6, no. 6, p. e21659, 2011.

[45] S. Shimada, K. Fukuda, and K. Hiraki, "Rubber Hand Illusion under Delayed Visual Feedback," PLoS ONE, vol. 4, no. 7, p. e6185, Jul. 2009.

[46] M. A. F. Ismail and S. Shimada, "Robot Hand Illusion under Delayed Visual Feedback: Relationship between the Senses of Ownership and Agency," PLOS ONE, vol. 11, no. 7, p. e0159619, Jul. 2016.

[47] S. Scheggi, L. Meli, C. Pacchierotti, and D. Prattichizzo, "Touch the virtual reality: using the leap motion controller for hand tracking and wearable tactile devices for immersive haptic rendering," in ACM SIGGRAPH 2015 Posters. ACM, 2015, p. 31.

[48] F. Weichert, D. Bachmann, B. Rudak, and D. Fisseler, "Analysis of the Accuracy and Robustness of the Leap Motion Controller," Sensors (Basel, Switzerland), vol. 13, no. 5, pp. 6380-6393, May 2013.

[49] L. Meli, S. Scheggi, C. Pacchierotti, and D. Prattichizzo, "Wearable haptics and hand tracking via an rgb-d camera for immersive tactile experiences," in ACM SIGGRAPH 2014 Posters. ACM, 2014, p. 56.

[50] C. Pacchierotti, F. Chinello, M. Malvezzi, L. Meli, and D. Prattichizzo, "Two Finger Grasping Simulation with Cutaneous and Kinesthetic Force Feedback," in Haptics: Perception, Devices, Mobility, and Communication. Springer, Berlin, Heidelberg, Jun. 2012, pp. 373-382.

[51] O. Christ and M. Reiner, "Perspectives and possible applications of the rubber hand and virtual hand illusion in non-invasive rehabilitation: Technological improvements and their consequences," Neuroscience \& Biobehavioral Reviews, vol. 44, pp. 33-44, Jul. 2014.

[52] H. H. Ehrsson, "Touching a Rubber Hand: Feeling of Body Ownership Is Associated with Activity in Multisensory Brain Areas," Journal of Neuroscience, vol. 25, no. 45, pp. 10 564-10 573, Nov. 2005.

[53] R. E. Kass and A. E. Raftery, "Bayes Factors," Journal of the American Statistical Association, vol. 90, no. 430, pp. 773-795, Jun. 1995.

[54] P. Beckerle, G. Salvietti, R. Unal, D. Prattichizzo, S. Rossi, C. Castellini, S. Hirche, H. Endo, S. Ben Amor, F. Ciocarlie, M. Mastrogiovanni, B. Argall, and M. Bianchi, "A human-robot interaction perspective on assistive and rehabilitation robotics," Frontiers in Neurorobotics, vol. 11, 2017.

[55] A. Marotta, M. Tinazzi, C. Cavedini, M. Zampini, and M. Fiorio, "Individual Differences in the Rubber Hand Illusion Are Related to Sensory Suggestibility," PLoS ONE, vol. 11, no. 12, Dec. 2016. [Online]. Available: http://www.ncbi.nlm.nih.gov/pmc/articles/ PMC5158054/

[56] D. Hecht and M. Reiner, "Sensory dominance in combinations of audio, visual and haptic stimuli," Experimental Brain Research, vol. 193, no. 2, pp. 307-314, Feb. 2009. [Online]. Available: http://link.springer.com/10.1007/s00221-008-1626-z 
[57] L. Shams and R. Kim, "Crossmodal influences on visual perception," Physics of Life Reviews, vol. 7, no. 3, pp. 269-284, Sep. 2010. [Online]. Available: http://linkinghub.elsevier.com/ retrieve/pii/S1571064510000424

[58] "The american heritage science dictionary," Jun 2017. [Online]. Available: http://www.dictionary.com/browse/proprioception

[59] J. Zhang, K. Ma, and B. Hommel, "The virtual hand illusion is moderated by context-induced spatial reference frames," Frontiers in Psychology, vol. 6, Oct. 2015.

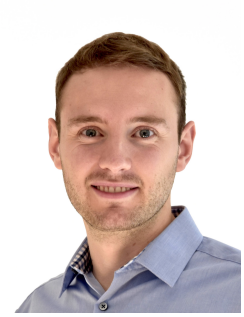

Jakob Fröhner received the M.S. degree in Psychology from the Technical University of Darmstadt, Darmstadt, Germany, in 2016. After finishing his master degree in psychology, he worked in a scholarship with Prof. Domenico Prattichizzo and Prof. Gionata Salvietti in the Department of Information Engineering in Siena Robotics and Systems Lab, University of Siena. Furthermore, he worked as a graduate assistant with Dr. Philipp Beckerle in the Institute for Mechatronic Systems in Mechanical Engineering at TUDarmstadt. He is now working with Prof. Sandra Hirche at the Chair of Information-Oriented Control at the Technical University of Munich, Munich, Germany as a research associate.

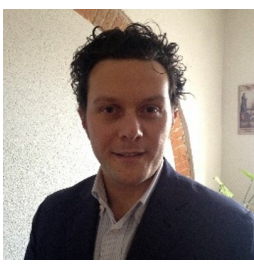

Gionata Salvietti (M12) received the M.S. degree in Robotics and Automation and the Ph.D. degree in Information Engineering from the University of Siena, Siena, Italy, in 2009 and 2012, respectively. He was a post-doc researcher with the Istituto Italiano di Tecnologia from 2012 to 2015. He is currently Assistant Professor at Department of Information Engineering and Mathematics, University of Siena and Research Affiliate at Dept. of Advanced Robotics at Istituto Italiano di Tecnologia. From 2016, Associate Editor IEEE Robotics and Automation Letters. His research interests are telemanipulation, robotic and human grasping, haptics and assistive devices.

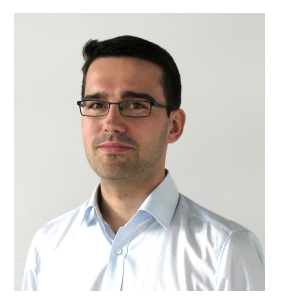

Philipp Beckerle (M12) received his Dr.-Ing in mechatronics from Technische Universität Darmstadt, Germany, in 2014. He is assistant professor in Elastic Lightweight Robotics at Technische Universität Dortmund and adjunct researcher at TU Darmstadt. His dissertation was awarded the Manfred-Hirschvogel Award 2015 and the MINT Excellence PhD thesis award 2015, he is recipient of the EugenHartmann Award 2017, and received an Athene Young Investigator grant in 2017. He was visiting researcher at Robotics \& Multibody Mechanics research group Vrije Universiteit Brussel, Interactive Robotics Lab and Human-Oriented Robotics and Control Lab, Arizona State University, as well as Human Centered Robotics Group, University of Siena. Dr. Beckerle takes over review and editorial responsibilities for various international journals and conferences. His main research topics are humanmachinecentered design, elastic actuation, wearable robotics control, and human-computer/robot interaction.

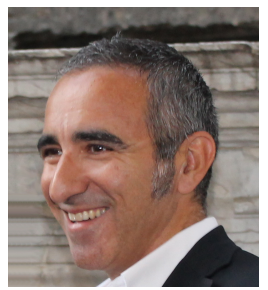

Domenico Prattichizzo (F16) received the Ph.D. degree in Robotics and Automation from the University of Pisa in 1995. Since 2002 he is an Associate Professor of Robotics at the University of Siena and since 2009 he is a Scientific Consultant at Istituto Italiano di Tecnoloogia. In 1994, he was a Visiting Scientist at the MIT Al Lab. Since 2014, he is Associate Editor of Frontiers on Robotics and Al. From 2007 to 2013 he has been Associate Editor in Chief of the IEEE Transactions on Haptics. From 2003 to 2007 he has been Associate Editor of the IEEE Transactions on Robotics and IEEE Transactions on Control Systems Technologies. He has been Chair of the Italian Chapter of the IEEE RAS (2006-2010), awarded with the IEEE 2009 Chapter of the Year Award. Research interests are in haptics, grasping, visual servoing, mobile robotics and geometric control. He is currently the Coordinator of the IP collaborative project WEARable HAPtics for Humans and Robots (WEARHAP). He is a fellow of the IEEE. 